\title{
Some Properties of Convolved $k$-Fibonacci Numbers
}

\author{
José L. Ramírez \\ Instituto de Matemáticas y sus Aplicaciones, Universidad Sergio Arboleda, Bogotá, Colombia \\ Correspondence should be addressed to José L. Ramírez; josel.ramirez@ima.usergioarboleda.edu.co
}

Received 30 April 2013; Accepted 28 May 2013

Academic Editors: S. Falcon and Y. Zhang

Copyright (c) 2013 José L. Ramírez. This is an open access article distributed under the Creative Commons Attribution License, which permits unrestricted use, distribution, and reproduction in any medium, provided the original work is properly cited.

We define the convolved $k$-Fibonacci numbers as an extension of the classical convolved Fibonacci numbers. Then we give some combinatorial formulas involving the $k$-Fibonacci and $k$-Lucas numbers. Moreover we obtain the convolved $k$-Fibonacci numbers from a family of Hessenberg matrices.

\section{Introduction}

Fibonacci numbers and their generalizations have many interesting properties and applications to almost every field of science and art (e.g., see [1]). The Fibonacci numbers $F_{n}$ are the terms of the sequence $0,1,1,2,3,5, \ldots$, wherein each term is the sum of the two previous terms, beginning with the values $F_{0}=0$ and $F_{1}=1$.

Besides the usual Fibonacci numbers many kinds of generalizations of these numbers have been presented in the literature. In particular, a generalization is the $k$-Fibonacci numbers.

For any positive real number $k$, the $k$-Fibonacci sequence, say $\left\{F_{k, n}\right\}_{n \in \mathbb{N}}$, is defined recurrently by

$$
\begin{gathered}
F_{k, 0}=0, \quad F_{k, 1}=1, \\
F_{k, n+1}=k F_{k, n}+F_{k, n-1}, \quad n \geq 1 .
\end{gathered}
$$

In [2], $k$-Fibonacci numbers were found by studying the recursive application of two geometrical transformations used in the four-triangle longest-edge (4TLE) partition. These numbers have been studied in several papers; see [27].

The convolved Fibonacci numbers $F_{j}^{(r)}$ are defined by

$$
\left(1-x-x^{2}\right)^{-r}=\sum_{j=0}^{\infty} F_{j+1}^{(r)} x^{j}, \quad r \in \mathbb{Z}^{+} .
$$

If $r=1$ we have classical Fibonacci numbers. Note that

$$
F_{m+1}^{(r)}=\sum_{j_{1}+j_{2}+\cdots+j_{r}=m} F_{j_{1}+1} F_{j_{2}+1} \cdots F_{j_{r}+1} \text {. }
$$

Moreover, using a result of Gould [8, page 699] on Humbert polynomials (with $n=j, m=2, x=1 / 2, y=-1, p=-r$, and $C=1$ ), we have

$$
F_{j+1}^{(r)}=\sum_{k=0}^{\lfloor j / 2\rfloor}\left(\begin{array}{c}
j+r-k-1 \\
j-k
\end{array}\right)\left(\begin{array}{c}
j-k \\
k
\end{array}\right) .
$$

It seems that convolved Fibonacci numbers first appeared in the classical Riordan's book [9]. These numbers have been studied in several papers; see [10-12]. In this paper, we obtain new identities for convolved $k$-Fibonacci numbers.

\section{Some Properties of $k$-Fibonacci Numbers and $k$-Lucas Numbers}

The characteristic equation associated with the recurrence relation (1) is $x^{2}=k x+1$. The roots of this equation are

$$
r_{1}=\frac{k+\sqrt{k^{2}+4}}{2}, \quad r_{2}=\frac{k-\sqrt{k^{2}+4}}{2} .
$$

Then we have the following basic identities:

$$
\begin{gathered}
r_{1}+r_{2}=k, \quad r_{1}-r_{2}=\sqrt{k^{2}+4}, \\
r_{1} r_{2}=-1 .
\end{gathered}
$$


TABLE 1: $F_{1, n}^{(r)}$, with $r=1,2,3,4,5,6,7,8 \ldots$

\begin{tabular}{|c|c|c|c|c|c|c|c|c|}
\hline$n$ & $F_{1, n}^{(1)}$ & $F_{1, n}^{(2)}$ & $F_{1, n}^{(3)}$ & $F_{1, n}^{(4)}$ & $F_{1, n}^{(5)}$ & $F_{1, n}^{(6)}$ & $F_{1, n}^{(7)}$ & $F_{1, n}^{(8)}$ \\
\hline 0 & 0 & 0 & 0 & 0 & 0 & 0 & 0 & 0 \\
\hline 1 & 1 & 1 & 1 & 1 & 1 & 1 & 1 & 1 \\
\hline 2 & 1 & 2 & 3 & 4 & 5 & 6 & 7 & 8 \\
\hline 3 & 2 & 5 & 9 & 14 & 20 & 27 & 35 & 44 \\
\hline 4 & 3 & 10 & 22 & 40 & 65 & 98 & 140 & 192 \\
\hline 5 & 5 & 20 & 51 & 105 & 190 & 315 & 490 & 726 \\
\hline 6 & 8 & 38 & 111 & 256 & 511 & 924 & 1554 & 2472 \\
\hline 7 & 13 & 71 & 233 & 594 & 1295 & 2534 & 4578 & 7776 \\
\hline$\vdots$ & $\vdots$ & $\vdots$ & $\vdots$ & $\vdots$ & $\vdots$ & $\vdots$ & $\vdots$ & $\vdots$ \\
\hline
\end{tabular}

TABLE 2: $F_{2, n}^{(r)}$, with $r=1,2,3,4,5,6,7,8, \ldots$

\begin{tabular}{|c|c|c|c|c|c|c|c|c|}
\hline$n$ & $F_{2, n}^{(1)}$ & $F_{2, n}^{(2)}$ & $F_{2, n}^{(3)}$ & $F_{2, n}^{(4)}$ & $F_{2, n}^{(5)}$ & $F_{2, n}^{(6)}$ & $F_{2, n}^{(7)}$ & $F_{2, n}^{(8)}$ \\
\hline 0 & 0 & 0 & 0 & 0 & 0 & 0 & 0 & 0 \\
\hline 1 & 1 & 1 & 1 & 1 & 1 & 1 & 1 & 1 \\
\hline 2 & 2 & 4 & 6 & 8 & 10 & 12 & 14 & 16 \\
\hline 3 & 5 & 14 & 27 & 44 & 65 & 90 & 119 & 152 \\
\hline 4 & 12 & 44 & 104 & 200 & 340 & 532 & 784 & 1104 \\
\hline 5 & 29 & 131 & 366 & 810 & 1555 & 2709 & 4396 & 6756 \\
\hline 6 & 70 & 376 & 1212 & 3032 & 6482 & 12432 & 22008 & 36624 \\
\hline 7 & 169 & 1052 & 3842 & 10716 & 25235 & 52808 & 101220 & 181224 \\
\hline$\vdots$ & $\vdots$ & $\vdots$ & $\vdots$ & $\vdots$ & $\vdots$ & $\vdots$ & $\vdots$ & $\vdots$ \\
\hline
\end{tabular}

Some of the properties that the $k$-Fibonacci numbers verify are summarized below (see $[2,6]$ for the proofs).

(i) Binet formula: $F_{k, n}=\left(r_{1}^{n}-r_{2}^{n}\right) /\left(r_{1}-r_{2}\right)$.

(ii) Combinatorial formula: $F_{k, n}=\sum_{i=0}^{\lfloor(n-1) / 2\rfloor}\left(\begin{array}{c}n-1-i \\ i\end{array}\right) \times$ $k^{n-1-2 i}$.

(iii) Generating function: $f_{k}(x)=x /\left(1-k x-x^{2}\right)$.

Definition 1. For any positive real number $k$, the $k$-Lucas sequence, say $\left\{L_{k, n}\right\}_{n \in \mathbb{N}}$, is defined recurrently by

$$
\begin{aligned}
& L_{k, 0}=2, \quad L_{k, 1}=k, \\
& L_{k, n+1}=k L_{k, n}+L_{k, n-1} .
\end{aligned}
$$

If $k=1$ we have the classical Lucas numbers. Some properties that the $k$-Lucas numbers verify are summarized below (see [13] for the proofs).

(i) Binet formula: $L_{k, n}=r_{1}^{n}+r_{2}^{n}$.

(ii) Relation with $k$-Fibonacci numbers: $L_{k, n}=F_{k, n-1}+$ $F_{k, n+1}, n \geq 1$.

\section{Convolved $k$-Fibonacci Numbers}

Definition 2. The convolved $k$-Fibonacci numbers $F_{k, j}^{(r)}$ are defined by

$$
f_{k}^{(r)}(x)=\left(1-k x-x^{2}\right)^{-r}=\sum_{j=0}^{\infty} F_{k, j+1}^{(r)} x^{j}, \quad r \in \mathbb{Z}^{+} .
$$

Note that

$$
F_{k, m+1}^{(r)}=\sum_{j_{1}+j_{2}+\cdots+j_{r}=m} F_{k, j_{1}+1} F_{k, j_{2}+1} \cdots F_{k, j_{r}+1} .
$$

Moreover, from Humbert polynomials (with $n=j, m=$ $2, x=k / 2, y=-1, p=-r$, and $C=1$ ), we have

$$
F_{k, j+1}^{(r)}=\sum_{l=0}^{\lfloor j / 2\rfloor}\left(\begin{array}{c}
j+r-l-1 \\
j-l
\end{array}\right)\left(\begin{array}{c}
j-l \\
l
\end{array}\right) k^{j-2 l} .
$$

If $r=1$ we obtain the combinatorial formula of $k$-Fibonacci numbers. In Tables 1,2 , and 3 some values of convolved $k$ Fibonacci numbers are provided. The purpose of this paper is to investigate the properties of these numbers.

Theorem 3. The following identities hold:

$$
\begin{aligned}
& \text { (1) } F_{k, 2}^{(r)}=k r, \\
& \text { (2) } F_{k, n}^{(r)}=F_{k, n}^{(r-1)}+k F_{k, n-1}^{(r)}+F_{k, n-2}^{(r)}, n \geq 2, \\
& \text { (3) } n F_{k, n+1}^{(r)}=r\left(k F_{k, n}^{(r+1)}+2 F_{k, n-1}^{(r+1)}\right), n \geq 1 .
\end{aligned}
$$

Proof. (1) Taking $j=1$ in (10), we obtain

$$
F_{k, 2}^{(r)}=\left(\begin{array}{l}
r \\
1
\end{array}\right)\left(\begin{array}{l}
1 \\
0
\end{array}\right) k=r k .
$$

(2) This identity is obtained from observing that

$$
\sum_{j=0}^{\infty} F_{k, j+1}^{(r)} x^{j}=\left(k x+x^{2}\right) \sum_{j=0}^{\infty} F_{k, j+1}^{(r)} x^{j}+\sum_{j=0}^{\infty} F_{k, j+1}^{(r-1)} x^{j} .
$$


TABLE 3: $F_{3, n}^{(r)}$, with $r=1,2,3,4,5,6,7,8 \ldots$

\begin{tabular}{|c|c|c|c|c|c|c|c|c|}
\hline$n$ & $F_{3, n}^{(1)}$ & $F_{3, n}^{(2)}$ & $F_{3, n}^{(3)}$ & $F_{3, n}^{(4)}$ & $F_{3, n}^{(5)}$ & $F_{3, n}^{(6)}$ & $F_{3, n}^{(7)}$ & $F_{3, n}^{(8)}$ \\
\hline 0 & 0 & 0 & 0 & 0 & 0 & 0 & 0 & 0 \\
\hline 1 & 1 & 1 & 1 & 1 & 1 & 1 & 1 & 1 \\
\hline 2 & 3 & 6 & 9 & 12 & 15 & 18 & 21 & 24 \\
\hline 3 & 10 & 29 & 57 & 94 & 140 & 195 & 259 & 332 \\
\hline 4 & 33 & 126 & 306 & 600 & 1035 & 1638 & 2436 & 3456 \\
\hline 5 & 109 & 516 & 1491 & 3385 & 6630 & 11739 & 19306 & 30006 \\
\hline 6 & 360 & 2034 & 6813 & 17568 & 38493 & 75348 & 135702 & 229176 \\
\hline 7 & 1189 & 7807 & 29737 & 85826 & 207935 & 445718 & 872130 & 1589664 \\
\hline : & $\vdots$ & $\vdots$ & : & : & $\vdots$ & : & : & $\vdots$ \\
\hline
\end{tabular}

(3) Taking the first derivative of $f_{k}^{(r)}(x)=\left(1-k x-x^{2}\right)^{-r}$, we obtain

$$
\begin{aligned}
\left(f_{k}^{(r)}(x)\right)^{\prime} & =\sum_{j=1}^{\infty} F_{k, j+1}^{(r)} j x^{j-1} \\
& =r\left(\frac{1}{1-k x-x^{2}}\right)^{r-1}\left(\frac{k+2 x}{\left(1-k x-x^{2}\right)^{2}}\right) \\
& =r(k+2 x) f_{k}^{(r+1)}(x) .
\end{aligned}
$$

Therefore the identity is clear.

In the next theorem we show that the convolved $k$ Fibonacci numbers can be expressed in terms of $k$-Fibonacci and $k$-Lucas numbers. This theorem generalizes Theorem 4 of [11].

Theorem 4. Let $j \geq 0$ and $r \geq 1$. We have

$$
\begin{aligned}
F_{k, j+1}^{(r)}= & \sum_{\substack{l=0 \\
r+l \cong 0 \bmod 2}}^{r-1}\left(\begin{array}{c}
r+l-1 \\
l
\end{array}\right)\left(\begin{array}{c}
r-l+j-1 \\
j
\end{array}\right) \\
& \times \frac{1}{\left(k^{2}+4\right)^{(r+l) / 2}} L_{k, r+j-l}^{(r)} \\
& +\sum_{\substack{l=0 \\
r+l \cong 1 \bmod 2}}^{r-1}\left(\begin{array}{c}
r+l-1 \\
l
\end{array}\right)\left(\begin{array}{c}
r-l+j-1 \\
j
\end{array}\right) \\
& \times \frac{1}{\left(k^{2}+4\right)^{(r+l-1) / 2}} F_{k, r+j-l}^{(r)} .
\end{aligned}
$$

Proof. Given $\alpha, \beta \in \mathbb{C}$, such that $\alpha \beta \neq 0$ and $\alpha \neq \beta$. Then we have the following partial fraction decomposition:

$$
\begin{aligned}
(1-\alpha z)^{-r}(1-\beta z)^{-r}= & \sum_{l=0}^{r-1}\left(\begin{array}{c}
-r \\
l
\end{array}\right) \frac{\alpha^{r} \beta^{l}}{(\alpha-\beta)^{r+l}}(1-\alpha z)^{l-r} \\
& +\sum_{l=0}^{r-1}\left(\begin{array}{c}
-r \\
l
\end{array}\right) \frac{\beta^{r} \alpha^{l}}{(\beta-\alpha)^{r+l}}(1-\beta z)^{l-r},
\end{aligned}
$$

where $\left(\begin{array}{l}t \\ 0\end{array}\right)=1$ and $\left(\begin{array}{l}t \\ l\end{array}\right)=(t(t-1) \cdots(t-l+1)) / l$ ! with $t \in \mathbb{R}$. Using the Taylor expansion

$$
(1-z)^{t}=\sum_{j=0}^{\infty}(-1)^{j}\left(\begin{array}{l}
t \\
j
\end{array}\right) z^{j}
$$

Then $(1-\alpha z)^{-r}(1-\beta z)^{-r}=\sum_{j=0}^{\infty} \gamma(j) z^{j}$, where

$$
\begin{aligned}
\gamma(j)= & \sum_{l=0}^{r-1}\left(\begin{array}{c}
-r \\
l
\end{array}\right) \frac{\alpha^{r} \beta^{l}}{(\alpha-\beta)^{r+l}}(-1)^{j}\left(\begin{array}{c}
l-r \\
j
\end{array}\right) \alpha^{j} \\
& +\sum_{l=0}^{r-1}\left(\begin{array}{c}
-r \\
l
\end{array}\right) \frac{\beta^{r} \alpha^{l}}{(\beta-\alpha)^{r+l}}(-1)^{j}\left(\begin{array}{c}
l-r \\
j
\end{array}\right) \beta^{j} .
\end{aligned}
$$

Note that $1-k z-z^{2}=\left(1-r_{1} z\right)\left(1-r_{2} z\right)$. On substituting these values of $\alpha=r_{1}$ and $\beta=r_{2}$ and using the identities (6), we obtain

$$
\begin{aligned}
F_{k, j+1}^{(r)}= & \sum_{l=0}^{r-1}\left(\begin{array}{c}
-r \\
l
\end{array}\right) \frac{(-1)^{l} \alpha^{r-l}}{\left(k^{2}+4\right)^{(r+l) / 2}}(-1)^{j}\left(\begin{array}{c}
l-r \\
j
\end{array}\right) \alpha^{j} \\
& +\sum_{l=0}^{r-1}\left(\begin{array}{c}
-r \\
l
\end{array}\right) \frac{(-1)^{l} \beta^{r-l}}{\left(k^{2}-4\right)^{(r+l) / 2}}(-1)^{j}\left(\begin{array}{c}
l-r \\
j
\end{array}\right) \beta^{j} \\
= & \sum_{l=0}^{r-1}(-1)^{l}\left(\begin{array}{c}
-r \\
l
\end{array}\right)(-1)^{j}\left(\begin{array}{c}
l-r \\
j
\end{array}\right) \\
& \times \frac{1}{\left(k^{2}+4\right)^{(r+l) / 2}}\left(\alpha^{r+j-l}+(-1)^{r+l} \beta^{r+j-l}\right) .
\end{aligned}
$$

Since that $(-1)^{l}\left(\begin{array}{c}-r \\ l\end{array}\right)=\left(\begin{array}{c}r+l-1 \\ l\end{array}\right)$ and $(-1)^{j}\left(\begin{array}{c}l-r \\ j\end{array}\right)=\left(\begin{array}{c}r-l+j-1 \\ j\end{array}\right)$, then

$$
\begin{aligned}
F_{k, j+1}^{(r)}= & \left(\begin{array}{c}
r+l-1 \\
l
\end{array}\right)\left(\begin{array}{c}
r-l+j-1 \\
j
\end{array}\right) \\
& \times \frac{1}{\left(k^{2}+4\right)^{(r+l) / 2}}\left(\alpha^{r+j-l}+(-1)^{r+l} \beta^{r+j-l}\right) .
\end{aligned}
$$

From the above equality and Binet formula, we obtain (14). 


\section{Hessenberg Matrices and Convolved $k$-Fibonacci Numbers}

An upper Hessenberg matrix, $A_{n}$, is an $n \times n$ matrix, where $a_{i, j}=0$ whenever $i>j+1$ and $a_{j+1, j} \neq 0$ for some $j$. That is, all entries below the superdiagonal are 0 but the matrix is not upper triangular:

$$
A_{n}=\left(\begin{array}{cccccc}
a_{1,1} & a_{1,2} & a_{1,3} & \cdots & a_{1, n-1} & a_{1, n} \\
a_{2,1} & a_{2,2} & a_{2,3} & \cdots & a_{2, n-1} & a_{2, n} \\
0 & a_{3,2} & a_{3,3} & \cdots & a_{3, n-1} & a_{3, n} \\
\vdots & \vdots & \vdots & \cdots & \vdots & \vdots \\
0 & 0 & 0 & \cdots & a_{n-1, n-1} & a_{n-1, n} \\
0 & 0 & 0 & \cdots & a_{n, n-1} & a_{n, n}
\end{array}\right) .
$$

We consider a type of upper Hessenberg matrix whose determinants are $k$-Fibonacci numbers. Some results about Fibonacci numbers and Hessenberg can be found in [14]. The following known result about upper Hessenberg matrices will be used.

Theorem 5. Let $a_{1}, p_{i, j}(i \leq j)$ be arbitrary elements of $a$ commutative ring $R$, and let the sequence $a_{1}, a_{2}, \ldots$ be defined by

$$
a_{n+1}=\sum_{i=1}^{n} p_{i, n} a_{i}, \quad(n=1,2, \ldots) .
$$

If

$$
A_{n}=\left(\begin{array}{cccccc}
p_{1,1} & p_{1,2} & p_{1,3} & \cdots & p_{1, n-1} & p_{1, n} \\
-1 & p_{2,2} & p_{2,3} & \cdots & p_{2, n-1} & p_{2, n} \\
0 & -1 & p_{3,3} & \cdots & p_{3, n-1} & p_{3, n} \\
\vdots & \vdots & \vdots & \cdots & \vdots & \vdots \\
0 & 0 & 0 & \cdots & a_{n-1, n-1} & a_{n-1, n} \\
0 & 0 & 0 & \cdots & -1 & a_{n, n}
\end{array}\right)
$$

then

$$
a_{n+1}=a_{1} \operatorname{det} A_{n} .
$$

In particular, if

$$
F_{n}^{(k)}=\left(\begin{array}{cccccc}
k & 1 & 0 & \cdots & 0 & 0 \\
-1 & k & 1 & \cdots & 0 & 0 \\
0 & -1 & k & \cdots & 0 & 0 \\
\vdots & \vdots & \vdots & \cdots & \vdots & \vdots \\
0 & 0 & 0 & \cdots & k & 1 \\
0 & 0 & 0 & \cdots & -1 & k
\end{array}\right)
$$

then from Theorem 5 we have that

$$
\operatorname{det} F_{n}^{(k)}=F_{k, n+1}, \quad(n=1,2, \ldots) .
$$

It is clear that the principal minor $M^{(k)}(i)$ of $F_{n}^{(k)}$ is equal to $F_{k, i} F_{k, n-i+1}$. It follows that the principal minor $M^{(k)}\left(i_{1}, i_{2}, \ldots, i_{l}\right)$ of the matrix $F_{n}^{(k)}$ is obtained by deleting rows and columns with indices $1 \leq i_{1}<i_{2}<\cdots<i_{l} \leq n$ :

$$
M^{(k)}\left(i_{1}, i_{2}, \ldots, i_{l}\right)=F_{k, i_{1}} F_{k, i_{2}-i_{1}} \cdots F_{k, i_{l}-i_{l-1}} F_{k, n-i_{l}+1} .
$$

Then we have the following theorem.
Theorem 6. Let $S_{n-l}^{(k)},(l=0,1,2, \ldots, n-1)$ be the sum of all principal minors of $F_{n}^{(k)}$ or order $n-l$. Then

$$
\begin{aligned}
S_{n-l}^{(k)} & =\sum_{j_{1}+j_{2}+\cdots+j_{l+1}=n-l} F_{k, j_{1}+1} F_{k, j_{2}+1} \cdots F_{k, j_{l+1}+1} \\
& =F_{k, n-l+1}^{(l+1)} .
\end{aligned}
$$

Since the coefficients of the characteristic polynomial of a matrix are, up to the sign, sums of principal minors of the matrix, then we have the following.

Corollary 7. The convolved $k$-Fibonacci number $F_{k, n-l+1}^{(l+1)}$ is equal, up to the sign, to the coefficient of $t^{l}$ in the characteristic polynomial $p_{n}(t)$ of $F_{n}^{(k)}$.

Corollary 8. The following identity holds:

$$
F_{k, n-l+1}^{(l+1)}=\sum_{i=0}^{\lfloor(n-l) / 2\rfloor}\left(\begin{array}{c}
n-i \\
i
\end{array}\right)\left(\begin{array}{c}
n-2 i \\
l
\end{array}\right) k^{n-2 i-l} .
$$

Proof. The characteristic matrix of $F_{n}^{(k)}$ has the form

$$
\left(\begin{array}{cccccc}
t-k & 1 & 0 & \cdots & 0 & 0 \\
-1 & t-k & 1 & \cdots & 0 & 0 \\
0 & -1 & t-k & \cdots & 0 & 0 \\
\vdots & \vdots & \vdots & \cdots & \vdots & \vdots \\
0 & 0 & 0 & \cdots & t-k & 1 \\
0 & 0 & 0 & \cdots & -1 & t-k
\end{array}\right)
$$

Then $p_{n}(t)=f_{n+1}(t-k)$, where $f_{n+1}(t)$ is a Fibonacci polynomial. Then from Corollary 8 and the following identity for Fibonacci polynomial [5]:

$$
f_{n+1}(x)=\sum_{i=0}^{\lfloor n / 2\rfloor}\left(\begin{array}{c}
n-i \\
i
\end{array}\right) x^{n-2 i}
$$

we obtain that

$$
\begin{gathered}
f_{n+1}(t-k)=\sum_{i=0}^{\lfloor n / 2\rfloor}\left(\begin{array}{c}
n-i \\
i
\end{array}\right) \sum_{l=0}^{n-2 i}\left(\begin{array}{c}
n-2 i \\
l
\end{array}\right) \\
\times(-1)^{n-l} k^{n-2 i-l} t^{l} .
\end{gathered}
$$

Therefore the corollary is obtained.

\section{Acknowledgments}

The author would like to thank the anonymous referees for their helpful comments. The author was partially supported by Universidad Sergio Arboleda under Grant no. USA-II2012-14.

\section{References}

[1] T. Koshy, Fibonacci and Lucas Numbers with Applications, Wiley-Interscience,, New York, NY, USA, 2001. 
[2] S. Falcón and Á. Plaza, "On the Fibonacci $k$-numbers," Chaos, Solitons and Fractals, vol. 32, no. 5, pp. 1615-1624, 2007.

[3] C. Bolat and H. Köse, "On the properties of $k$-Fibonacci numbers," International Journal of Contemporary Mathematical, vol. 5, no. 22, pp. 1097-1105, 2010.

[4] S. Falcon, "The $k$-Fibonacci matrix and the Pascal matrix," Central European Journal of Mathematics, vol. 9, no. 6, pp. 14031410, 2011.

[5] S. Falcón and Á. Plaza, "On $k$-Fibonacci sequences and polynomials and their derivatives," Chaos, Solitons and Fractals, vol. 39, no. 3, pp. 1005-1019, 2009.

[6] S. Falcón and Á. Plaza, "The $k$-Fibonacci sequence and the Pascal 2-triangle," Chaos, Solitons and Fractals, vol. 33, no. 1, pp. 38-49, 2007.

[7] A. Salas, "About $k$-Fibonacci numbers and their associated numbers," International Mathematical Forum, vol. 50, no. 6, pp. 2473-2479, 2011.

[8] H. W. Gould, "Inverse series relations and other expansions involving Humbert polynomials," Duke Mathematical Journal, vol. 32, no. 4, pp. 697-711, 1965.

[9] J. Riordan, Combinatorial Identities, Wiley, New York, NY, USA, 1968.

[10] V. E. Hoggatt Jr. and M. Bicknell-Johnson, "Fibonacci convolution sequences," Fibonacci Quarterly, vol. 15, no. 2, pp. 117-122, 1977.

[11] P. Moree, "Convoluted convolved Fibonacci numbers," Journal of Integer Sequences, vol. 7, article 04.2.2, 2004.

[12] G. Liu, "Formulas for convolution Fibonacci numbers and polynomials," Fibonacci Quarterly, vol. 40, no. 4, pp. 352-357, 2002.

[13] S. Falcon, "On the k-Lucas numbers," International Journal of Contemporary Mathematical, vol. 6, no. 21, pp. 1039-1050, 2011.

[14] M. Janjić, "Hessenberg matrices and integer sequences," Journal of Integer Sequences, vol. 13, no. 7, pp. 1-10, 2010. 


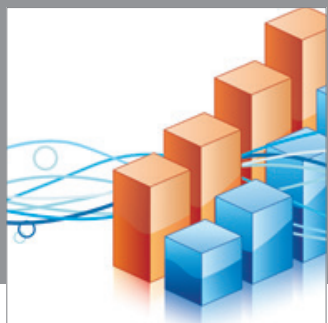

Advances in

Operations Research

mansans

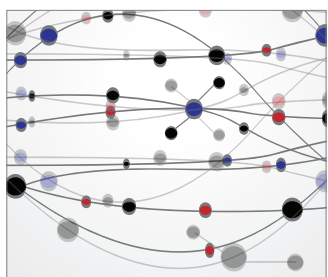

The Scientific World Journal
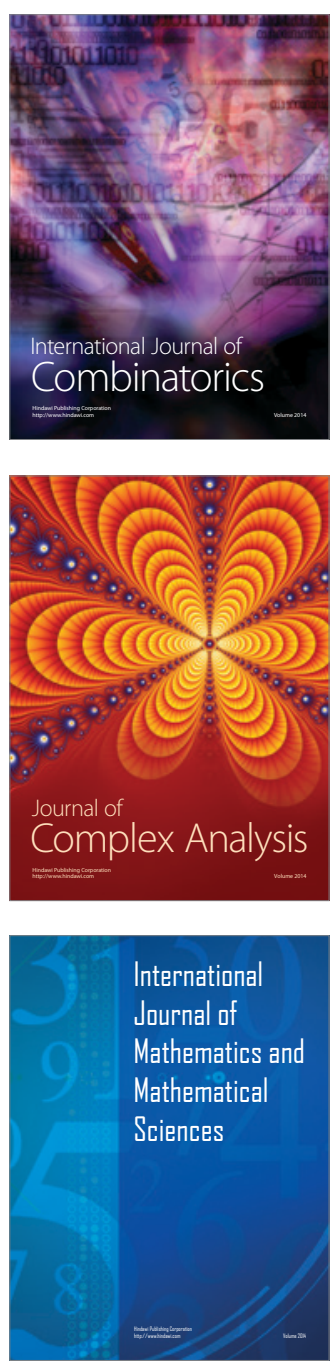
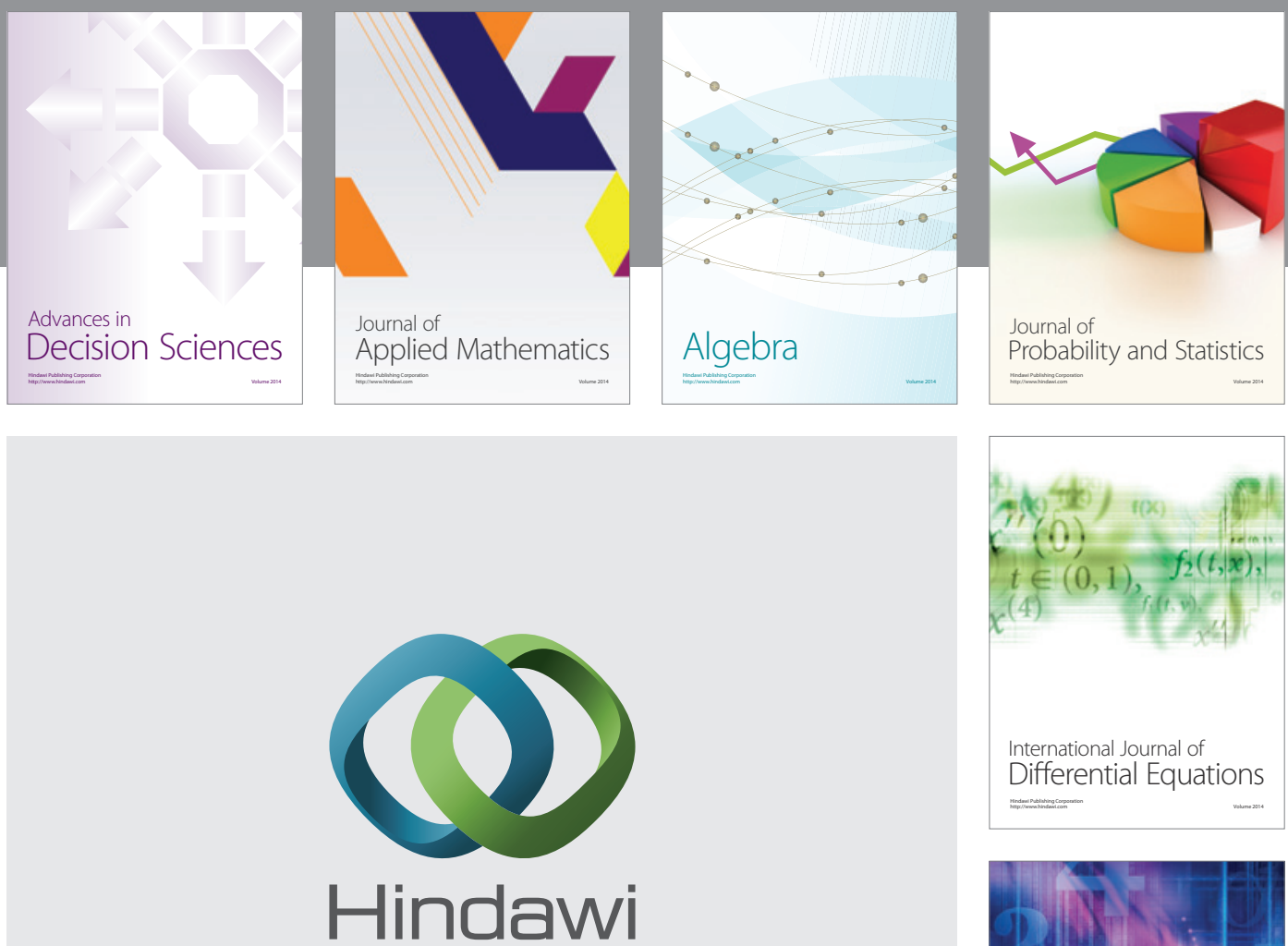

Submit your manuscripts at http://www.hindawi.com
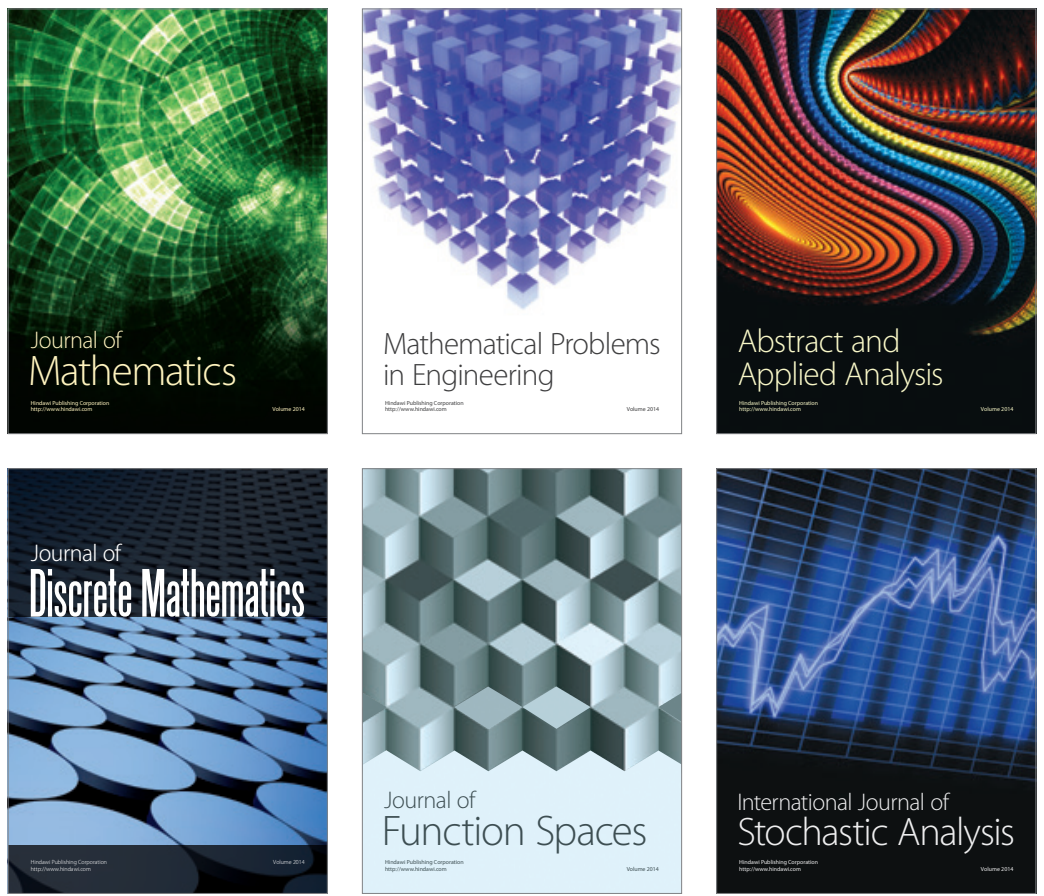

Journal of

Function Spaces

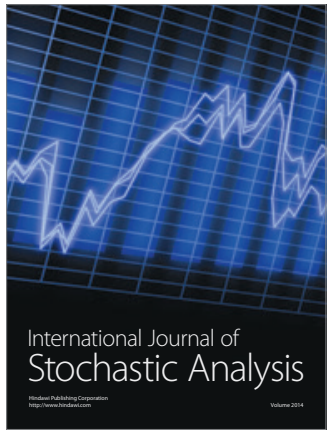

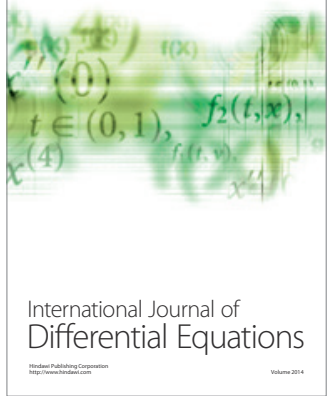
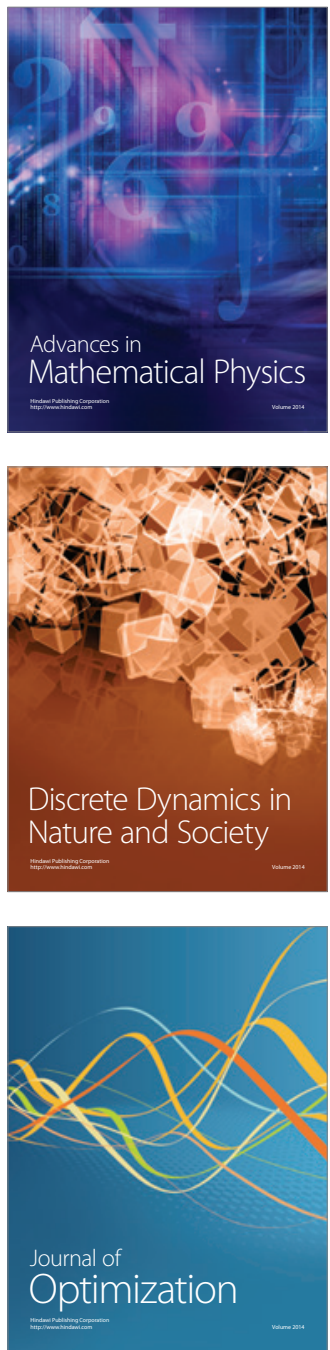